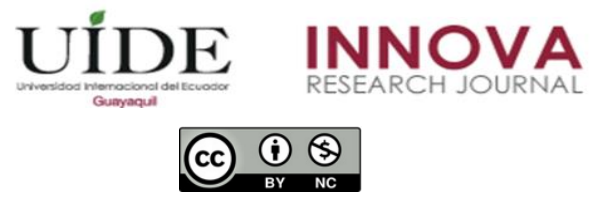

INNOVA Research Journal, ISSN 2477-9024

(Septiembre-Diciembre, 2019). Vol. 4, No.3 pp. 13-22

DOI: https://doi.org/10.33890/innova.v4.n3.2019.969

URL: http://revistas.uide.edu.ec/index.php/innova/index

Correo: innova@uide.edu.ec

\title{
Evaluación del Modelo de Dimensiones del Aprendizaje Organizacional - DLOQ
}

\section{Evaluation of the Organizational Learning Dimensions Model - DLOQ}

Carlos Manosalvas Vaca

Luis Manosalvas Vaca

Universidad Estatal Amazónica, Ecuador

Roxanna Palma León

Universidad Federal de Uberlandia, Brasil

Cecilia Aguirre

Escuela Superior Politécnica de Chimborazo, Ecuador

Autor para correspondencia: cmanosalvas@uea.edu.ec; rpalmaleon@ufu.br; lmanosalvas@uea.edu.ec; ceci28a@hotmail.com

Fecha de recepción: 12 de marzo de 2019 - Fecha de aceptación: 24 de junio del 2019

Resumen: El aprendizaje organizacional es uno de los conceptos de mayor relevancia en los últimos años ya que permite la generación de ventajas competitivas para una organización. Sin embargo su operacionalización y medición es objeto de debate a nivel teórico y empírico. En esta investigación se realizó un análisis de confiabilidad y validez a través de un análisis factorial confirmatorio del modelo de Dimensiones del Aprendizaje Organizacional propuesto por Marsick y Watkins, uno de los cuestionarios y modelos más utilizados en varios contextos pero que aún ha sido aplicado muy poco en economías emergentes. Se encuestó a muestra representativa de trabajadores de empresas de tecnología y los resultados permitieron concluir que el instrumento cumple con los criterios de confiablidad, validez convergente y divergente, no obstante no mostró un buen ajuste con los datos.

Palabras Clave: aprendizaje organizacional; factorial confirmatorio

Abstract: Organizational learning is one of the most relevant concepts in recent years. It allows the generation of competitive advantages for an organization. However, its operationalization and measurement is the subject of theoretical and empirical debate. In this research, an analysis of reliability and validity was carried out through a confirmatory factorial analysis of Dimenssions of Learning Organization Questionnaire proposed by Marsick and Watkins was carried out, one of the most used instruments in several contexts but not in emerging economies. A representative sample of workers from technology companies was surveyed and the results allowed to conclude that the instrument meets the criteria of reliability, convergent and divergent validity, however it did not show a good fit with the data.

Key Words: organizational learning; confirmatory factor 


\section{Introducción}

En la actualidad, aspectos como la globalización y el desarrollo sin precedentes de las tecnologías de la información y comunicación, que proveen un nivel de conectividad sin precedentes (Maswera, Dawson \& Edwards, 2008), han generado un contexto de constante cambio que obliga a las empresas a analizar y rediseñar sus estrategias constantemente, con la finalidad de crear ventajas competitivas que posibiliten su éxito al mediano y largo plazo. En un esfuerzo por reforzar su dirección estratégica, mejorar su rendimiento y apoyar los procesos de gestión de cambios, las organizaciones en todas las esferas de la actividad comercial y social continúan buscando enfoques viables para el uso adecuado de datos y la generación de conocimiento (Belle, 2016). Con base en esto, el campo del aprendizaje organizacional ha sido uno de los conceptos que ha captado la atención de investigadores y gerentes ya que provee mecanismos mediante los cuales se desarrollan capacidades y conocimientos para obtener ventajas competitivas (Crossan y Berdrow, 2003) así como la capacidad de detectar, interpretar y responder a la información interna y externa (Chiva y Alegre, 2005). De acuerdo a Imran, IIyas y Aslam (2016) en el entorno empresarial actual altamente volátil, el aprendizaje organizacional se ha convertido en la fuente de ventaja competitiva para organizaciones dinámicas.

No obstante, a pesar de la importancia que tiene esta variable para el desempeño organizacional y la creación de ventajas competitivas, en la literatura académica existe una confusión conceptual en este campo que de alguna manera afecta los estudios empíricos relacionados (Jyothibabu, Farooq \& Bhusan Pradhan, 2010). Muchos investigadores han desarrollado escalas para medir el aprendizaje organizacional (Bontis, Crossan \& Hulland., 2002; Tippins y Sohi, 2003; Templeton, Lewis \& Snyder., 2002). Sin embargo, aún no se ha desarrollado una escala de medición adecuada (Jyothibabu, Farooq \& Bhusan Pradhan, 2010). De acuerdo Watkins y Dirani (2003) los especialistas en desarrollo organizacional y de talento humano han tenido pocas medidas organizacionales válidas para demostrar el estado del aprendizaje y el impacto del aprendizaje en la organización.

Con base en esto, en este artículo se realizó el análisis del Cuestionario de las Dimensiones de una organización orientada al aprendizaje (DLOQ por sus siglas en Inglés) propuesto por Watkins y Dirani (2003) el cual permite evaluar y construir una arquitectura de aprendizaje autosostenible que se inserte en la cultura e incluso en el trabajo mismo de cada individuo de la organización y que evoluciona y crece con la misma. Para conocimiento de los autores de esta investigación este instrumento no ha sido implementado en economías emergentes y existen muy pocos estudios desarrollados con una traducción al español del mismo. El objetivo es aplicar un análisis factorial confirmatorio que permita verificar la validez divergente y convergente del instrumento propuesto por Marsick y Watkins (2003) en empresas que realizan sus actividades en economías emergentes.

En los siguientes apartados se realizará una breve revisión de la literatura académica relacionada a la variable de estudio para posteriormente explicar la metodología y resultados obtenidos. Al final se especificarán las conclusiones más importantes y futuros estudios que podrían implementarse. 


\section{Revisión de la Literatura}

El aprendizaje organizacional, definido como el proceso a través del cual se crea conocimiento organizacional extrayendo nuevo conocimiento, desde el conocimiento actual (García-Morales, Jiménez-Barrionuevo \& Gutiérrez-Gutiérrez, 2012) puede abordarse desde dos puntos de vista: como un comportamiento organizacional y desde una perspectiva estratégica y operacional (Yeung, Lai \& Yee, 2007). Desde la perspectiva de comportamiento organizacional, el aprendizaje en la organización ocurre a través del aprendizaje individual, y cuando el proceso de aprender está incrustado entre todos los miembros de la organización, es decir, cuando se establece una cultura de aprendizaje (Iebra, Zegarra \& Zegarra, 2011). Desde un punto de vista estratégico, una organización orientada al aprendizaje se define como una organización experta en la creación, adquisición e intercambio de conocimiento, y en la modificación de su comportamiento con el objetivo de reflejar nuevos conocimientos y perspectivas (Garvin, 1993). En ambas perspectivas está implícita la noción de un clima orientado al aprendizaje

En la literatura relacionada al aprendizaje organizacional, se encuentran varias definiciones que están íntimamente relacionadas. Las dos más citadas y analizadas son: aprendizaje organizacional y organización orientada al aprendizaje. La primera, hace referencia a los procesos y actividades relacionadas al aprendizaje que ocurren dentro de una organización, analizándolas de forma descriptiva (Amy, 2008), y desde un punto de vista académico (Olsson, 2015). Por otro lado, la literatura relacionada a la organización orientada al aprendizaje es de naturaleza prescriptiva y está enfocada hacía gerentes y consultores (Amy, 2007). Sin embargo, ambos enfoques guardan estrecha relación, principalmente, en tres áreas: el papel de la alineación con el medio ambiente, la necesidad del aprendizaje individual y su transferencia a nivel organizacional, y la prioridad de maximizar el impacto de factores contextuales como la estrategia, estructura y cultura. Bajo este contexto, en el presente estudio, ambas definiciones tendrán el mismo significado. Además, en este estudio se abordará el aprendizaje organizacional desde el punto de vista del comportamiento

El aprendizaje organizacional permite obtener un desempeño superior cuando es valorado por la gerencia y cuando el conocimiento es compartido en rutinas organizacionales a través de una infraestructura apropiada y una cultura de aprendizaje (Yeung, Lai \& Yee, 2007). Esta cultura de aprendizaje puede configurarse como una capacidad dinámica ya que de acuerdo a Teece (2007) las capacidades dinámicas permiten que una organización obtenga una ventaja competitiva a través de la creación, despliegue y protección de activos intangibles y no transferibles que respaldan un desempeño organizacional superior. Pavlou y El Sawy (2011) argumentaron además que las capacidades dinámicas permiten obtener un mejor desempeño organizacional al igual que Wang, Senaratne y Rafiq (2015) para quienes este tipo de capacidades son fundamentales para el rendimiento diferencial de las empresas.

Con base en los expuesto, dos perspectivas principales han surgido en el desarrollo de una escala para la medición del aprendizaje organizacional (Chiva et al., 2007). La primera busca la presencia de habilitadores de aprendizaje en la organización a través de su cuestionario. La segunda perspectiva busca resultados de aprendizaje en la organización (Jyothibabu, Farooq \& Bhusan Pradhan, 2010). El instrumento desarrollado por Marsick y Watkins (2003) se enmarca en la primera perspectiva pues busca construir una arquitectura de aprendizaje autosostenible tan 
arraigada en la cultura e incluso en el propio trabajo que evoluciona y crece con la organización. Un diagnóstico válido y confiable permite a los profesionales del desarrollo organizacional evaluar dónde se encuentra la cultura de aprendizaje en el momento de su administración, y señala el camino para implementar los cambios necesarios que permitan desarrollar una infraestructura de aprendizaje más efectiva. Este instrumento mide la percepción que tienen los miembros de una organización sobre las siete dimensiones que debe tener una organización que fomenta un adecuado clima de aprendizaje: (a) crear en forma continua oportunidades de aprendizaje, (b) promover la investigación y el diálogo, (c) fomentar la colaboración y el aprendizaje en equipo, (d) crear sistemas para capturar y compartir el aprendizaje, (e) empoderar a la gente hacía una visión colectiva, (f) conectar a la organización con su entorno y (g) proporcionar un liderazgo estratégico para el aprendizaje.

\section{Metodología}

La metodología de investigación fue de tipo transversal no experimental, y se utilizaron fuentes de datos primarias y secundarias. Los encuestados fueron lo que Drucker (1998) denominó "Trabajadores del Conocimiento", definidos como personas que utilizan su conocimiento como principal herramienta de trabajo. Toffler (1990) identificó a un típico trabajador del conocimiento, como aquel que se desempeña como científico, ingeniero o una persona que opera una tecnología sofisticada; además, este tipo de trabajador debe ser capaz de crear y mejorar su conocimiento tecnológico o gestionar el conocimiento tecnológico de sus compañeros de trabajo.

Bajo este contexto, la población de estudio estuvo conformada por profesionales que laboran en empresas de tecnología, donde los procesos de gestión de conocimiento y aprendizaje son fundamentales para desarrollar su trabajo. Para la selección de los elementos de la muestra, se tomó como referencia el Ranking Empresarial de EKOS (2016), el cual incluye un total de 5000 compañías que operan en diversos sectores. De este ranking, se seleccionaron las empresas que ofrecen servicios tecnológicos (clasificación J del CIIU).

La técnica de muestreo utilizada fue un muestreo por conveniencia y estratificado, en función del rubro tecnológico al que se dedica la empresa (tecnologías de información o telecomunicaciones). Del universo de 5000 compañías del Ranking empresarial de EKOS se seleccionaron todas las empresas categorizadas en los rubros Tecnologías de la Información (23 empresas) y Telecomunicaciones (41 empresas), sumando un total de 64 empresas. Con el objetivo de obtener la máxima tasa de respuesta se decidió aplicar el estudio a todas las compañías seleccionadas. Las encuestas estuvieron aplicadas a los empleados de cada una de estas empresas.

El instrumento fue validado por expertos académicos y profesionales en el área de gestión de conocimiento y aprendizaje organizacional. Además fue sometido a un proceso de doble traducción para verificar la consistencia del contenido de las preguntas. Una vez realizadas estas validaciones, se procedió a contactar por vía telefónica y correo electrónico, a cada uno de los gerentes de las 64 empresas seleccionadas, solicitando su apertura para desarrollar el estudio. Se recibió respuesta de tan solo 6 empresas, a quienes se les hizo llegar una versión On line de los 
cuestionarios a través del área de recursos humanos, para que sea aplicada a todos los empleados y a los gerentes de nivel intermedio, recibiendo un total de 269 encuestas válidas.

La validez de cada instrumento de medida se evaluó a través de un Análisis Factorial Confirmatorio. Las etapas a desarrollar en esta parte del análisis fueron: (a) identificación del modelo, (b) ajuste del modelo, (c) validez convergente y (d) validez discriminante. Un modelo de medida puro para hacer este tipo de análisis, es aquel cuyas variables latentes tienen indicadores observables y donde cada variable latente covaría con las otras variables latentes (Kline, 2005; Silva \& Sheiner, 2005). El análisis factorial confirmatorio debe aplicarse para instrumentos de medida que han sido completamente desarrollados y cuya estructura factorial ha sido validada (Byrne, 2009).

\section{Resultados}

\section{Confiabilidad}

La confiabilidad de una escala, se define como la proporción de la varianza atribuible a la puntuación verdadera de la variable latente (DeVellis, 2003). Lo que se busca es determinar la consistencia interna de las escalas, es decir, la homogeneidad de los ítems dentro de la escala, lo cual se puede hacer calculando el Alpha de Cronbach, un coeficiente que, de acuerdo a Ajzen (2007), debe ser mayor o igual a 0.7 para considerar una escala confiable, mientras que, valores mayores a 0.5 pueden considerarse cuestionables y valores menores a este último se consideran ya inaceptables. Los índices obtenidos de cada uno de los subconstructos fueron mayores a 0.7 por lo que se puede concluir que las escalas son confiables.

\section{Análisis Factorial Confirmatorio}

La identificación del modelo, la cual pudo lograrse siguiendo los criterios de Jarvis, Mackenzie y Podsakoff (2003): (a) en la escala de medida de cada variable latente se debe restringir uno de los caminos de esta variable a un valor de uno, (b) los caminos o enlaces de los errores de las variables observables y de los errores de las variables endógenas latentes se restringieron a un valor de uno. Una vez lograda la identificación de los modelos, su ajuste fue evaluado con índices mínimos que, según Kline (2016) deben ser reportados: (a) prueba chicuadrado (con p-value $>0.20$ ), (b) índice residual de la raíz cuadrada media estandarizada (SRMR), (c) índice de ajuste comparativo (CFI) y (d) el índice de la raíz cuadrada media del error de aproximación (RMSEA). Los valores límite para cada uno de estos índices se tomaron de acuerdo a las sugerencias de Byrne (2009).

La validez convergente y divergente se evaluó siguiendo el procedimiento establecido por Anderon y Gerbing (1998). La validez de convergencia se determinó evaluando si el coeficiente estimado de cada indicador sobre su constructo subyacente es significativo (mayor al doble de su error estándar). La validez discriminante fue evaluada restringiendo el parámetro de correlación entre dos variables latentes a 1 , y realizando una prueba de diferencias chi cuadrado entre el modelo restringido y el modelo sin restricción; las pruebas se hicieron por pares de constructos a la vez. 
El constructo de Aprendizaje Organizacional utilizado en la presente investigación, se mide a través de 7 subconstructos y un total de 43 variables observables. Siguiendo procedimientos similares a los demás constructos, se determinó el ajuste y la validez convergente y discriminante del modelo de medida. En la Figura 1 se observa el modelo probado. Al hacer un primer análisis del modelo, este mostró un ajuste muy pobre, con valores de SRMR igual a 0.067 y CFI de 0.771. Al revisar los índices de modificación, se pudo apreciar que el ajuste mejoraría si se especifican covarianzas entre los errores de variables observables que miden aspectos similares dentro del mismo constructo; una vez especificadas, el ajuste del modelo mejoró, sin embargo, no se llegó a los valores esperados, por lo que se puede concluir que este modelo de medida no presenta un buen ajuste.

Tabla 1 Índices de Ajuste del Modelo Clima de Aprendizaje Organizacional

\begin{tabular}{ccc}
\hline Índice & Valor & Nivel de Aceptación \\
\hline Chi-cuadrado & 1890.160 & $\mathrm{P}<0.00$ \\
Gl & 830 & \\
SRMR & 0.047 & $\leq 0.05$ \\
CFI & 0.85 & $\geq 0.95$ \\
RMSEA & 0.0978 & $\leq 0.06$ \\
CMIN/DF & 3.58 & $<5$ \\
\hline
\end{tabular}

El modelo de medida también fue sometido a pruebas de normalidad obteniendo un coeficiente de Mardia igual a 65.47, mientras que los valores individuales de curtosis de cada variable estuvieron por debajo de 7 (Byrne, 2009) lo que indica que los datos pueden considerarse con distribución normal. La validez convergente del modelo quedó demostrada al corroborar que todos los estimados de regresión entre las variables observables y su respectiva variable latente, fueron significativas, es decir, su valor estimado fue mayor al doble del error estándar; además, todos los parámetros estandarizados son mayores a 0.5. En la Tabla 2 se puede ver un extracto de estos indicadores La Tabla 3 muestra la prueba de diferencias entre el modelo restringido y sin restricción para cada par de variables latentes. Estas diferencias fueron significativas demostrando así la validez discriminante del modelo de medida del constructo Clima de Aprendizaje Organizacional. 


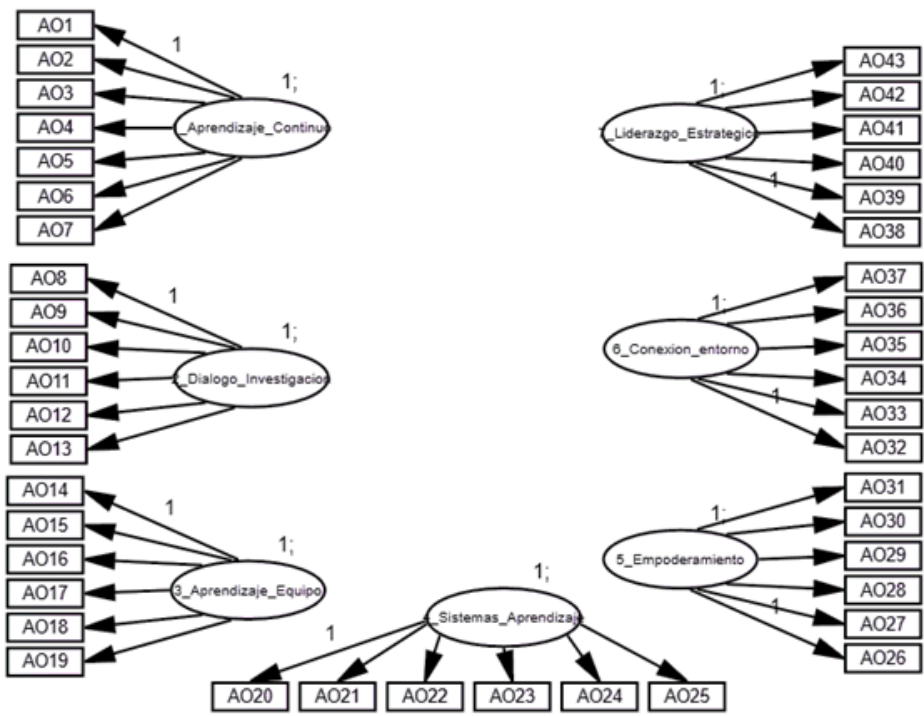

Figura 1. Modelo de Medida Simplificado del Aprendizaje Organizacional sin correlaciones entre subconstructos

Tabla 2. Pesos de Regresión entre variables observables y constructo de Aprendizaje Organizacional

\begin{tabular}{|c|c|c|c|c|c|c|c|}
\hline & & Relación & $\begin{array}{c}\text { Peso } \\
\text { Estandarizado }\end{array}$ & $\begin{array}{c}\text { Peso } \\
\text { Estandarizado }\end{array}$ & $\begin{array}{c}\text { Error } \\
\text { Estándar }\end{array}$ & $\mathrm{CR}$ & $\begin{array}{l}\text { p- } \\
\text { valué }\end{array}$ \\
\hline $\mathrm{AO} 7$ & $\begin{array}{ll}<-- \\
\end{array}$ & Aprendizaje Continuo & 1.000 & 0.610 & & & \\
\hline AO6 & $<---$ & Aprendizaje Continuo & 1.138 & 0.759 & 0.113 & 10.054 & $* * *$ \\
\hline $\mathrm{AO} 5$ & $<---$ & Aprendizaje Continuo & 0.997 & 0.677 & 0.108 & 9.249 & $* * *$ \\
\hline $\mathrm{AO} 4$ & $<---$ & Aprendizaje Continuo & 0.803 & 0.531 & 0.085 & 9.501 & $* * *$ \\
\hline $\mathrm{AO} 3$ & $<--$ & Aprendizaje Continuo & 1.150 & 0.781 & 0.112 & 10.261 & $* * *$ \\
\hline $\mathrm{AO} 2$ & $<--$ & Aprendizaje Continuo & 0.977 & 0.711 & 0.102 & 9.585 & $* * *$ \\
\hline $\mathrm{AO} 1$ & $<---$ & Aprendizaje Continuo & 0.963 & 0.648 & 0.08 & 8.940 & $* * *$ \\
\hline $\mathrm{AO} 43$ & $<---$ & Liderazgo & 1.000 & 0.714 & & & \\
\hline $\mathrm{AO} 42$ & $<--$ & Liderazgo & 1.305 & 0.856 & 0.096 & 13.594 & $* * *$ \\
\hline $\mathrm{AO} 41$ & $<--$ & Liderazgo & 1.331 & 0.860 & 0.097 & 13.667 & $* * *$ \\
\hline $\mathrm{AO} 40$ & $<--$ & Liderazgo & 1.199 & 0.806 & 0.094 & 12.790 & $* * *$ \\
\hline AO39 & $<--$ & Liderazgo & 1.168 & 0.739 & 0.100 & 11.715 & $* * *$ \\
\hline $\mathrm{AO} 38$ & $<--$ & Liderazgo & 1.333 & 0.852 & 0.098 & 13.532 & $* * *$ \\
\hline AO13 & $<--$ & Diálogo_Investigación & 1.000 & 0.817 & & & \\
\hline $\mathrm{AO} 12$ & $<--$ & Diálogo Investigación & 0.644 & 0.633 & 0.058 & 11.094 & $* * *$ \\
\hline AO11 & $<---$ & Diálogo Investigación & 1.024 & 0.825 & 0.065 & 15.869 & $* * *$ \\
\hline $\mathrm{AO} 10$ & $<---$ & Diálogo Investigación & 1.045 & 0.733 & 0.078 & 13.421 & $* * *$ \\
\hline $\mathrm{AO} 9$ & $<---$ & Diálogo Investigación & 0.992 & 0.790 & 0.067 & 14.902 & $* * *$ \\
\hline $\mathrm{AO} 8$ & $<---$ & Diálogo Investigación & 0.986 & 0.779 & 0.067 & 14.619 & $* * *$ \\
\hline
\end{tabular}


Tabla 3. Comparación entre el Modelo Restringido y no Restringido Clima de Aprendizaje Organizacional

\begin{tabular}{lccc}
\hline & & & Diferencias \\
& Modelo sin restricciones & Modelo restringido & \\
& $(847 \mathrm{GL})$ & $(848 \mathrm{GL})$ & \\
\hline Aprendizaje Continuo - Diálogo e Investigación & 4511.697 & 4518.374 & 6.68 \\
Aprendizaje Continuo - Aprendizaje en Equipo & 4563.620 & 4568.393 & 4.773 \\
Aprendizaje Continuo - Sistemas de Aprendizaje & 4659.960 & 4665.332 & 5.372 \\
Aprendizaje Continuo - Empoderamiento & 4697.707 & 4705.266 & 7.559 \\
Aprendizaje Continuo - Conexión con Entorno & 4655.387 & 4662.267 & 6.88 \\
Aprendizaje Continuo - Liderazgo Estratégico & 4659.906 & 4670.905 & 10.99 \\
Diálogo e Investigación - Aprendizaje en Equipo & 4522.891 & 4528.979 & 6.088 \\
Diálogo e Investigación - Sistemas de & 4656.357 & 4663.755 & 7.398 \\
Aprendizaje & & & \\
Diálogo e Investigación - Empoderamiento & 4715.905 & 4727.882 & 11.977 \\
Diálogo e Investigación - Conexión con Entorno & 4669.749 & 4678.882 & 9.133 \\
Diálogo e Investigación - Liderazgo Estratégico & 4673.936 & 4688.833 & 14.897 \\
Aprendizaje en Equipo - Sistemas de Aprendizaje & 4658.969 & 4663.598 & 4.629 \\
Aprendizaje en Equipo - Empoderamiento & 4676.802 & 4682.143 & 5.341 \\
Aprendizaje en Equipo - Conexión con Entorno & 4634.706 & 4638.877 & 4.171 \\
Aprendizaje en Equipo - Liderazgo Estratégico & 4598.463 & 4604.811 & 6.348 \\
Sistemas de Aprendizaje - Empoderamiento & 4634.072 & 4638.696 & 4.624 \\
Sistemas de Aprendizaje - Conexión con Entorno & 4623.107 & 4627.264 & 4.157 \\
Sistemas de Aprendizaje - Liderazgo Estratégico & 4698.838 & 4710.083 & 11.245 \\
Empoderamiento - Conexión con Entorno & 4543.170 & 4547.167 & 3.997 \\
Empoderamiento - Liderazgo Estratégico & 4630.498 & 4637.354 & 6.856 \\
Conexión con Entorno - Liderazgo Estratégico & 4530.106 & 4533.990 & 3.884 \\
\hline & & & \\
\hline
\end{tabular}

\section{Discusión y Conclusiones}

Esta investigación buscó comprobar la validez del instrumento de medición del Aprendizaje Organizacional propuesto por Marsick y Watkins (2003) dentro de un contexto empresarial que desarrolla sus actividades en economías emergentes. El cuestionario fue aplicado a una muestra de empleados que trabajan en empresas que ofrecen servicios de tecnología.

Los resultados mostraron que el modelo de medida no tuvo un buen ajuste ya que los índices de bondad de ajuste en su mayoría no alcanzaron los umbrales requeridos. El análisis de 
los índices de modificación indicaron que se podría mejorar el ajuste si se correlacionaban ciertas errores de cada una de las variables no obstante, las correlaciones sugerían asociaciones entre subconstructos diferentes por lo que, futuras investigaciones deberán analizar a fondo el contenido de cada una de las preguntas con el objetivo de verificar si realmente están evaluando aspectos similares y si podrían combinarse o eliminarse del modelo, así como realizar la comprobación de los nuevos modelos obtenidos con el modelo original.

Por otro lado la validez convergente y divergente del modelo fue comprobada exitosamente lo que permite concluir que cada una de las preguntas mide adecuadamente los subconstructos correspondientes. Además, dada la evidencia de validez divergente se concluye que cada uno de los subconstructos no está correlacionados y son conceptos diferentes que en su conjunto permiten obtener una evaluación integral del Aprendizaje Organizacional. Futuros estudios deberán analizar si el instrumento se puede modelar como de primer o segundo orden así como su posible aplicación a otros sectores empresariales.

\section{Bibliografía}

Amy, A. (2008). Leaders as facilitators of individual and organizational learning. Leadership \& Organizational Development Journal, 29(3), 212-234.

Ajzen, I. (2007). Constructing a TpB questionnaire: Conceptual and methodological considerations. Recuperado de: http://www.people.umass.edu/aizen

Anderson, J. C. \& Gerbin, D. W. (1988). Structural equation modeling in practice: A review and recommended two-step approach. Psychological Bulleting, 100(3), 411-423.

Belle, S. (2016). Organizational learning? Look again. The Learning Organization, 23(5), 332341.

Bontis, N., Crossan, M. M., \& Hulland, J. (2002). Managing an organizational learning system by aligning stocks and flows. Journal of management studies, 39(4), 437-469.

Byrne, B. (2009). Structural Equation Modeling with Amos (2nd ed.). New York: NY, Taylor and Francis Group.

Chiva, R., \& Alegre, J. (2005). Organizational learning and organizational knowledge: towards the integration of two approaches. Management learning, 36(1), 49-68.

Crossan, M. M., \& Berdrow, I. (2003). Organizational learning and strategic renewal. Strategic management journal, 24(11), 1087-1105.

DeVilles, F. (2003). Scale Development, Theory and Application. Sage Publication

García-Morales, V. J., Jiménez-Barrionuevo, M. M. \& Gutiérrez-Gutiérrez, L. (2012). Transformational Leadership influence on organizational perfomance through organizational learning and innovation. Journal of Business Research, 65, 1040-1050

Garvin, D. A. (1993). Building a learning organization. Harvard Business Review, 7(4), 78-91.

Iebra, L., Zegarra, P. \& Zegarra, A. (2011). Learning for Sharing: an empirical analysis of organizational learning and knowledge sharing. International Entrepreneurship and Management Journal, 7(4), 509-518.

Imran, M. K., Ilyas, M., \& Aslam, U. (2016). Organizational learning through transformational leadership. The learning organization, 23(4), 232-248. 
Jarvis, C. B., Mackenzie, S. B., \& Podsakoff, P. M. (2003). A critical review of construct indicators and measurement model misspecification in marketing and consumer research. Journal of Consumer Research, 30, 199-218.

Jyothibabu, C., Farooq, A., \& Bhusan Pradhan, B. (2010). An integrated scale for measuring an organizational learning system. The Learning Organization, 17(4), 303-327.

Kline, R. B. (2016). Principles and practice of structural equation modeling (3rd ed.). New York, NY: Guilford.

Marsick, V. J., \& Watkins, K. E. (2003). Demonstrating the value of an organization's learning culture: the dimensions of the learning organization questionnaire. Advances in Developing Human Resources, 5(2), 132-151.

Maswera, T., Dawson, R. \& Edwards, J. (2008). E-Commerce Adoption of Travel and Tourism Organizations in South Africa, Kenya, Zimbabwe and Uganda. Telematics and Informatics, $25,187-200$.

Olsson, T. (2015). Eight steps to Sustainable Organizational Learning. Management for Professionals. DOI 10.1007/978-3-319-15937-9

Teece, D. J. (2007). Explicating dynamic capabilities: the nature and microfoundations of (sustainable) enterprise performance. Strategic Management Journal, 28(13), 1319-1350.

Templeton, G. F., Lewis, B. R., \& Snyder, C. A. (2002). Development of a measure for the organizational learning construct. Journal of management information systems, 19(2), 175218.

Tippins, M. J., \& Sohi, R. S. (2003). IT competency and firm performance: is organizational learning a missing link? Strategic management journal, 24(8), 745-761.

Wang, C. L., Senaratne, C., \& Rafiq, M. (2015). Success traps, dynamic capabilities and firm performance. British Journal of Management, 26(1), 26-44

Watkins, K. E., \& Dirani, K. M. (2013). A meta-analysis of the dimensions of a learning organization questionnaire: Looking across cultures, ranks, and industries. Advances in Developing Human Resources, 15(2), 148-162.

Yeung, A. C. L., Lai, K. H. \& Yee, R. W. Y. (2007). A measure of knowledge sharing behavior: Scale development and Validation. Knowledge Management Research and Practice, 7(1), 65-81. 